This is the author's final, peer-reviewed manuscript as accepted for publication. The publisher-formatted version may be available through the publisher's web site or your institution's library.

\title{
Enhancement of condensation heat transfer with patterned surfaces
}

Abhra Chatterjee, Melanie M. Derby, Yoav Peles, Michael K. Jensen

\section{How to cite this manuscript}

If you make reference to this version of the manuscript, use the following information:

Chatterjee, A., Derby, M. M., Peles, Y., \& Jensen, M. K. (2014). Enhancement of condensation heat transfer with patterned surfaces. Retrieved from http://krex.ksu.edu

\section{Published Version Information}

Citation: Chatterjee, A., Derby, M. M., Peles, Y., \& Jensen, M. K. (2014). Enhancement of condensation heat transfer with patterned surfaces. International Journal of Heat and Mass Transfer, 71, 675-681.

Copyright: $@ 2014$ Elsevier Ltd

Digital Object Identifier (DOI): doi:10.1016/j.jijheatmasstransfer.2013.12.069

Publisher's Link: http://www.sciencedirect.com/science/article/pii/S0017931014000027

This item was retrieved from the K-State Research Exchange (K-REx), the institutional repository of Kansas State University. K-REx is available at http://krex.ksu.edu 


\title{
Enhancement of Condensation Heat Transfer with Patterned Surfaces
}

\author{
Abhra Chatterjee, Melanie M. Derby, Yoav Peles, Michael K. Jensen \\ Department of Mechanical, Aerospace, and Nuclear Engineering \\ Rensselaer Polytechnic Institute \\ Troy, NY 12180
}

\begin{abstract}
An experimental study of condensation heat transfer on surfaces with patterns of distinct hydrophilic and hydrophobic regions has been carried out. The patterned surfaces of $25.4 \mathrm{~mm}$ diameter comprised of $25 \%$ hydrophilic region and $75 \%$ hydrophobic region by area. Experiments were performed on surfaces with several feature sizes and shapes of the patterns. The feature sizes varied from $0.25 \mathrm{~mm}$ to 1.50 mm. Two types of pattern shapes were studied: circular hydrophilic regions on an otherwise hydrophobic surface (island-patterns), and hydrophilic region resembling a tree on a background of hydrophobic region (tree-pattern). Depending on the type of pattern on the condensation surface, the heat transfer coefficients were either higher or lower than that of the completely hydrophobic surface. For the range of inlet vapor velocities (about $0.05 \mathrm{~m} / \mathrm{s}$ to $5 \mathrm{~m} / \mathrm{s}$ ), among all the surfaces, the highest heat transfer
\end{abstract}


coefficient was observed for the patterned surface with the feature size of $0.25 \mathrm{~mm}$, which was higher than that of a completely hydrophobic surface.

Keywords Condensation; pattern; hydrophilic; hydrophobic; feature size

\section{Introduction}

Among the most relevant areas of application of two-phase heat transfer are in the power generation industry, air-conditioning systems, cooling of electronic devices, and the distillation industry. For power generation the Rankine cycle, which evaporates and condenses the working fluid, is one of the most widely employed processes. In the electronics thermal management industry, devices are often cooled by boiling a fluid in a closed loop; after boiling the evaporated working fluid then has to be condensed back to liquid. In the distillation industry liquid is evaporated and then condensed back into liquid to remove dissolved salts and other impurities. Thus, in all the above mentioned industries condensation is an important process and enhancement of heat transfer coefficients will benefit a wide range of industries.

One of the most popular methods to enhance condensation heat transfer coefficients is through the modification of physical and chemical properties of the condensation surface $[1,2,3]$. For surfaces whose chemical and physical properties are modified, the interaction of the condensate with the condensation surface is also altered [2].

Condensation modes can be classified as filmwise condensation (FWC) and dropwise condensation (DWC) [1]. If the condensate forms a film on the condensation surface, it 
is termed FWC and if it is in the form of droplets, it is said to be DWC [1]. FWC, observed for surfaces with high surface energy, is associated with lower heat transfer coefficients when compared to that of DWC. Heat transfer coefficient is also enhanced with increasing mobility of the droplets [4]. The mobility of the condensate for DWC is better than for $F W C$ which is partly attributed to higher heat transfer coefficient for DWC when compared to FWC [5]. Droplet mobility can be improved by having a surface energy gradient on the condensation surface [4]. When a surface with a wettability gradient is used, droplets move from the region of lower wettability to regions of higher wettability [4].

Although hydrophobic surfaces promote $D W C$, resulting in higher heat transfer coefficients than hydrophilic surfaces [1], hydrophilic surfaces show higher rates of condensation when compared to hydrophobic surfaces [6]. To benefit from both surface characteristics, a pattern of hydrophilic and hydrophobic surfaces can be utilized on a single condensation surface, which would result in better mobility of the condensate than a uniform surface $[4,7]$. Therefore, to achieve high heat transfer coefficients, patterns of distinct hydrophobic and hydrophilic regions on the condensation surface can be used [8]. While some patterns can result in higher heat transfer coefficients than that of a hydrophobic surface, others can result in lower heat transfer coefficients [7]. Therefore, patterns with distinct hydrophobic and hydrophilic regions must be carefully designed with due importance to the shape and size of the pattern features. In the present study, condensation heat transfer on patterns with distinct hydrophobic and hydrophilic regions has been studied. 


\section{Experimental Apparatus and Procedure}

To obtain condensation heat transfer coefficients on a flat vertical surface, a flow loop (Fig. 1) was constructed to supply steam to a vapor chamber.

The loop consisted of a steam supply line, a filter, a steam separator, a valve, an electric immersion heater, a test section, a heat exchanger, a flow meter, and a drain. The entire flow loop was insulated to minimize heat loss.

The test section (Fig. 2) consisted of a vapor chamber, a piston, a vertical condensation surface, the test section, and a visualization window. Insulation was applied on the vapor chamber to reduce the heat loss. The vapor chamber was $124 \mathrm{~mm}$ long and $45.7 \mathrm{~mm}$ wide, and the depth of the chamber could be varied between $2 \mathrm{~mm}$ to $25 \mathrm{~mm}$. By varying the depth of the vapor chamber, the hydraulic diameter of the vapor chamber could be varied. The hydraulic diameter of the vapor chamber is defined as:

$$
D=4 A_{c} / P_{c}
$$

where, $D$ is the hydraulic diameter of the chamber, $A_{c}$ is the area of cross-section of the vapor chamber across which vapor flowed and $P_{c}$ is the perimeter of the same cross section.

The condensation surface was comprised of a 25.4-mm diameter, 7-mm thick cylindrical coupon made of oxygen-free copper (Alloy 101). One of the two flat sides of the cylindrical coupon served as the condensation surface and the other side was 
soldered to a copper block, which was cooled at the opposite end through a bath circulator, which maintained the cooling water temperature at a specified value.

Experiments were performed on various kinds of patterned surfaces. The patterned surfaces consisted of distinct hydrophilic and hydrophobic regions. For all the surfaces the proportion of the hydrophilic and the hydrophobic regions was maintained at $25 \%$ and $75 \%$ by area, respectively (Fig. 3). The only difference between the surfaces was in the feature sizes and in the shape of the patterns.

Experiments were performed on the following feature sizes and shapes of the patterns:

1. Pattern shape: island-patterns:

The island-patterns consisted of hydrophilic circular islands of different sizes on an otherwise hydrophobic surface. The various island diameters studied were:

a) Hydrophilic circular islands of $1.50 \mathrm{~mm}$ diameter;

b) Hydrophilic circular islands of $0.75 \mathrm{~mm}$ diameter;

c) Hydrophilic circular islands of $0.50 \mathrm{~mm}$ diameter;

d) Hydrophilic circular islands of $0.25 \mathrm{~mm}$ diameter;

2. Pattern shape: linear and connected hydrophilic regions, tree-pattern:

a) The branches in the tree-pattern were $1.50 \mathrm{~mm}$ wide

Details of the procedures for the preparation of the patterned surfaces have been described in [9]. 
For all the surfaces with island-patterns, the circular hydrophilic islands were arranged in concentric circles with equal spacing between the neighboring islands in the radial and the azimuthal directions. The distance between the edges of two neighboring hydrophilic islands for each of the feature sizes of the island-patterns is given in Table 1.

\section{Data Reduction}

To obtain data, the cooling water bath circulator was set to a temperature of $30{ }^{\circ} \mathrm{C}$, so that a constant temperature was maintained at the cooling end of the copper block. The mass flow rate of the cooling water was about $0.2 \mathrm{~kg} / \mathrm{s}$ and the change in the temperature of the cooling water was less than $0.3{ }^{\circ} \mathrm{C}$. Followed by the bath circulator, the steam supply was turned on, and the valve upstream of the test section was adjusted to obtain the desired mass flow. The immersion heater was then switched on and the heating power was adjusted with the help of a variable power supply to obtain a stable and small superheat of approximately of $0.5^{\circ} \mathrm{C}$ before the steam entered the test section. The superheat was necessary to obtain the exact state at which the vapor entered the test section and to calculate the quality of the steam leaving the test section through a heat balance. After steady state was reached, the temperature and pressure were recorded using a computer controlled data acquisition system of National Instruments signal chassis (SCXI-1000) and LabVIEW software. The flow parameters were then altered, and the entire procedure was repeated. The same steps were carried out for several positions of the piston with the hydraulic diameter of the vapor chamber ranging 
between $4.5 \mathrm{~mm}$ and $32.5 \mathrm{~mm}$. The data were reduced and analyzed with the help of computer programs written in Engineering Equations Solver (EES) software.

Visualization of the condensates on the condensation surface was done with a Nikon digital camera.

Temperature, pressure, and mass flow rate of the vapor and the temperatures in the copper block were directly measured and were used to calculate heat flux, heat transfer coefficient, wall subcooling, and exit quality of the fluid.

Five equally spaced thermocouples were used to obtain the temperature along the axis of the copper block. A linear curve fit of the temperature and the location of the thermocouples gave the temperature gradient along the axis of the copper block. Assuming 1-D heat transfer, Fourier's law was then used to calculate the heat flux through the test section:

$$
q^{\prime \prime}=-k_{c u} d T / d y
$$

where $q^{\prime \prime}$ is the heat flux through the condensation surface, $k_{c u}$ is the thermal conductivity of oxygen-free copper and $d T / d y$ is the temperature gradient along the copper block.

Another thermocouple fixed to the copper coupon and close to the condensation surface was used to calculate the wall temperature using the following equation: 


$$
T_{w}=T_{1}+q^{\prime \prime} \Delta y / k_{c u}
$$

where $T_{w}$ is the wall temperature, $T_{1}$ is the temperature reading of the thermocouple closest to the condensation surface, and $\Delta y$ is the distance between the thermocouple and the condensation surface.

The heat transfer coefficient was calculated using the following equation [10]:

$$
h=q^{\prime \prime} /\left(T_{\text {sat }}-T_{w}\right)
$$

where $h$ is the heat transfer coefficient and $T_{\text {sat }}$ is the saturation temperature of the vapor.

The temperature and pressure were measured with an accuracy of $\pm 0.1{ }^{\circ} \mathrm{C}$ and $5 \mathrm{kPa}$, respectively, while the steam flow rate was measured with an accuracy of $0.5 \mathrm{~mL} / \mathrm{min}$. The uncertainty analysis was carried out using the error-ratio and standard deviation method adapted by Kedzierski and Worthington [11]. The typical uncertainties in the calculated heat transfer coefficients were estimated to be less than $\pm 6 \%$.

\section{Results and Discussion}

First the results of the island-patterns on the condensation surface have been discussed followed by that of the tree-pattern. 


\subsection{Island-Patterns}

All the patterned surfaces with hydrophilic islands showed similar trends with regards to the heat transfer coefficient, the inlet vapor velocity and interaction of the condensate with the condensation surface. However, although the trends observed on the different surfaces were similar, the maximum heat transfer coefficients observed for each of the surfaces were different. Here, the patterned surface with hydrophilic island diameter of $0.75 \mathrm{~mm}$ has been taken as a representative case and is discussed in detail.

\subsubsection{Effect of Inlet Vapor Velocity on the Heat Transfer Coefficient}

For the range of steam inlet velocity and the hydraulic diameters of the experiment, the highest heat transfer coefficient observed was around $25900 \mathrm{~W} / \mathrm{m}^{2} \mathrm{~K}$ (Fig. 4). The hydraulic diameter of the vapor chamber showed an effect on the heat transfer coefficients, which was similar to that of completely hydrophobic surface, i.e., the mass flow rates for which the heat transfer coefficients leveled off was lower for smaller hydraulic diameters when compared to that of higher hydraulic diameters.

Visualization of the condensation modes showed that the hydrophilic regions were covered by bigger droplets while the hydrophobic region had relatively smaller droplets covering them (Fig. 5). This is because the hydrophilic surfaces have higher surface energies and show higher rates of condensation. Similar to the experimental study by Varanasi et al. [6]. Unlike the case of completely hydrophobic surface, steady streams or rivulets of condensates were not observed [10]. At lower vapor inlet velocities, it was 
observed that the droplets formed in the hydrophilic islands after growing to departure diameter were impeded by the neighboring hydrophobic region from moving vertically downward. However, this effect was much less pronounced at higher vapor velocities [10].

The droplets were seen to nucleate, grow, coalesce and depart from the nucleation sites, as shown in Fig 5. However, the droplets were distributed evenly over the surface and no steady rivulets were observed, unlike what was reported by Chatterjee et al. [12] and Baojin et al. [3] for a completely hydrophobic surface. This was because the droplets were preferentially formed on the hydrophilic islands which were distributed evenly on the surface [10].

It was reported by Mccormick and Baer [12] that the condensate droplet size and dynamics had a significant influence on the heat transfer coefficient. On the other hand, Grooten and Geld [13] reported that with higher frequency of droplet removal, the average size of droplets sticking onto the condensation surface decreased. The size to which the droplets could grow without infringing into the water repelling hydrophobic regions was restricted by the island hydrophilic island diameters as shown in Figs 5 and 6. For bigger island diameters, the droplets could grow to a larger diameter when compared to that of smaller island diameters. Also, the frequency with which the droplets departed from their respective condensation sites was observed to be lower for the surface with feature size of $1.50 \mathrm{~mm}$ than that of feature size of $0.75 \mathrm{~mm}$.

The higher frequency in the case of the island diameter of $0.25 \mathrm{~mm}$ when compared to that of the $1.5 \mathrm{~mm}$ was partly because of coalescence of the droplets for the island 
diameter of $0.25 \mathrm{~mm}$. The circular islands were $0.2 \mathrm{~mm}$ apart from edge to edge for the feature size of $0.25 \mathrm{~mm}$ and were $1.4 \mathrm{~mm}$ apart for the feature size of $1.5 \mathrm{~mm}$ (Table 1 ). Therefore, owing to the closer distance between the droplets, which primarily formed on the hydrophilic islands, the droplets could more easily coalesce for the feature size of $0.25 \mathrm{~mm}$, thereby resulting in higher departure frequency for the feature size of $0.25 \mathrm{~mm}$ when compared to $1.50 \mathrm{~mm}$.

The droplets departed in about $12 \mathrm{~s}$ for the island diameter of $1.50 \mathrm{~mm}$ where as for the island diameter of $0.75 \mathrm{~mm}$, the droplets departed every $9 \mathrm{~s}$. Thus, the decreasing heat transfer coefficients with increasing island feature sizes can be attributed to both the larger droplet departure diameters (increasing with increasing feature size) and the frequency of departure (decreasing with increasing feature size).

\subsubsection{Effect of hydrophilic Island Diameter on Heat Transfer Coefficients}

The highest heat transfer coefficients observed for the patterned surfaces with hydrophilic islands was around $34000 \mathrm{~W} / \mathrm{m}^{2} \mathrm{~K}, 29000 \mathrm{~W} / \mathrm{m}^{2} \mathrm{~K}, 26000 \mathrm{~W} / \mathrm{m}^{2} \mathrm{~K}$, and $22000 \mathrm{~W} / \mathrm{m}^{2} \mathrm{~K}$ for island diameters of $0.25 \mathrm{~mm}, 0.50 \mathrm{~mm}, 0.75 \mathrm{~mm}$, and $1.50 \mathrm{~mm}$, respectively (Fig. 7). It can be observed that the heat transfer coefficients increased with decreasing hydrophilic island diameters; this can be attributed to smaller droplets on the condensation surface resulting in higher heat transfer coefficients when compared to that of larger droplets on the condensation surface. Also, the frequency with which the droplets departed from their nucleation point was higher for smaller feature sizes. 


\subsection{Patterned Surface with Continuous Hydrophilic Avenues, Tree-Pattern}

The condensation surface with the pattern resembling a tree differed with the patterned hydrophilic islands in the fact that for the tree-pattern, the hydrophilic regions were linear and connected with each other. However, the proportion of the total area covered by hydrophilic region was the same as that of the patterns with islands. The connecting avenues of the tree-pattern were $1.50 \mathrm{~mm}$ wide.

\subsubsection{Effect of Inlet Vapor Velocity on Heat Transfer Coefficients}

The effect of inlet vapor velocity on the heat transfer coefficients for the tree-pattern condensation surface was similar to the completely hydrophobic surface and the patterned surface with hydrophilic islands. The highest heat transfer coefficient observed for the range of inlet vapor velocity and hydraulic diameter of the test section at a cooling water temperature of $30{ }^{\circ} \mathrm{C}$ was about $22500 \mathrm{~W} / \mathrm{m}^{2} \mathrm{~K}$ (Fig. 8).

The mass flow rate of the steam at which the heat transfer leveled off, also decreased with decreasing hydraulic diameter of the test section as was observed for the previously discussed modified surfaces.

Visualization of the interaction of the condensate with the condensation surface revealed nucleation and growth of droplets on a few peripheral regions of the surface and at the corners of the branches of the trees (Fig. 9). Some of the droplets formed in the hydrophobic regions were sucked into the hydrophilic branches of the tree-pattern, a large number of droplets were seen close to the edges of the condensation surface No steady stream of condensate was observed on the condensation surface. 


\subsection{Comparison of heat transfer coefficients for Different Surfaces}

Similar heat transfer experiments were performed on a surfaces without patterns, i.e., a completely hydrophilic surface and completely hydrophobic surface [14]. Comparing the experimental results from [14] and the discussions in this study, the highest heat transfer coefficients observed for all the modified condensation surfaces were higher than those of the completely hydrophilic surface (i.e., bare copper). The reason for this is the fact that the combination of DWC and FWC was observed on all the modified surface, while the hydrophilic surface showed only FWC.

The heat transfer coefficients were observed to depend mainly on the droplet size of the condensate and to a certain extent on the frequency of departure of the droplets from their nucleation sites. The highest heat transfer coefficients were observed for the modified condensation surface with hydrophilic island patterns of diameter of $0.25 \mathrm{~mm}$. Figure 11 shows the comparison of the highest heat transfer coefficients observed for all the surfaces on which experiments were performed.

Although higher heat transfer coefficients are observed with hydrophobic surfaces when compared to hydrophilic surfaces [2], patterns of distinct hydrophilic and hydrophobic regions have been observed to have enhanced heat transfer coefficients, as has been demonstrated by Leu et al. [7]. In the present case, all the patterned surfaces had a hydrophilic region, occupying $25 \%$ of the total condensation surface area, which can be linked to the lower heat transfer coefficients observed on some of the patterned surfaces when compared to the completely hydrophobic surface. However, the droplet 
sizes observed on the hydrophilic island diameter of $0.25 \mathrm{~mm}$ were even smaller than the droplets seen on the completely hydrophobic surfaces (Fig. 10). Also, the condensation rate was higher on the patterned condensation surface of feature size $0.25 \mathrm{~mm}$ as it offered at least $25 \%$ hydrophilic regions, which promote condensation, as compared to the completely hydrophobic surface. With a higher rate of condensation and smaller droplets, the condensation surface was washed more frequently by departing droplets, thus exposing new nucleation sites $[10,13,15]$. Also, no steady stream of condensate was observed on the patterned surface, whereas streams of condensate, which are predominantly $F W C$, were observed on the completely hydrophobic surface [10]. A combination of the above mentioned factors may have resulted in the higher heat transfer coefficients for the patterned condensation surface with island diameter of $0.25 \mathrm{~mm}$ when compared to those of the completely hydrophobic surface. The highest heat transfer coefficients observed for each of the condensation surfaces is given in Table 2 .

\section{Conclusions}

An experimental parametric study on enhancement of heat transfer coefficients was performed on modified surfaces, with patterned of distinct hydrophobic and hydrophilic regions. The conclusions drawn from the study are the following:

- The heat transfer coefficient was significantly affected by the inlet vapor velocity.

The highest heat transfer coefficient observed was around $34000 \mathrm{~W} / \mathrm{m}^{2} \mathrm{~K}$, which was around $7.5 \%$ higher than that of the completely hydrophobic surface. The highest 
heat transfer coefficient, for all the surfaces tested in this study, was obtained for the patterned surface with hydrophilic island diameter of $0.25 \mathrm{~mm}$.

- The highest heat transfer coefficient obtained for each of the patterned surfaces with island-patterns increased with decreasing island size for the range of island diameter studied.

- The mode of interaction of the condensate on the surface was observed to be a combination of dropwise condensation (DWC) and filmwise condensation (FWC). Droplets were mainly observed to nucleate and grow on the hydrophilic islands. The droplets departed from their nucleation points after coalescing with neighboring droplets.

- The increase in the heat transfer coefficient with decreasing hydrophilic island diameter in the island-patterns was because the island feature size determined the droplet departure diameter and the frequency of departure. Smaller islands ensured smaller droplets, easier coalescence, and higher departure frequency.

- Smaller droplet departure diameter and higher frequency of droplet departure helped enhance the heat transfer coefficient in the island-pattern with feature size of 0.25 mm when compared to that of the completely hydrophobic surface.

\section{Acknowledgments}

The authors acknowledge the financial support of Office of Naval Research through a MURI grant. Grant Number N00014-07-1-0723 entitled "System-Level Approach for Multi-Phase, Nanotechnology-Enhanced Cooling of High-Power Microelectronic 
Systems." Support and cooperation from Rensselaer Polytechnic Institute is also acknowledged.

\section{Nomenclature}

A

$A_{c}$

$D$

$h$

$k_{c u}$

$\dot{m}$

$P_{C}$

Q"

$d T / d y$

$T$

$T_{w}$

$T_{1}$

$\dot{V}$

y area of cross section of the copper block

area of cross section of vapor chamber across which vapor flowed

hydraulic diameter of the vapor chamber

heat transfer coefficient

thermal conductivity of oxygen free copper

vapor mass flow rate

perimeter of cross section of vapor chamber across which vapor flowed

heat flux

temperature gradient along the copper cooling block

absolute temperature

calculated wall temperature

temperature of the thermocouple closest to the condensation surface

volume flow rate of the condensed steam

distance of thermocouple

\section{Reference List}

[1] A. Faghri, Y. Zhang, J. Howell, Condensation and evaporation, Advanced Heat 
Transfer, first ed., Global Digital Press, Columbia, MO, 2010.

[2] Z. Dongchang, L. Zaiqi, L. Jifang, New surface materials for dropwise condensation, in: Proceedings of the 8th International Heat Transfer Conference, San Francisco, CA, 1986, pp. 1677-1682.

[3] Q. Baojin, Z. Li, X. Hong, S. Yan, Experimental study on condensation heat transfer of steam on vertical titanium plates with different surface energies, Experimental Thermal and Fluid Science, 35 (1) (2011) 211-218.

[4] R.W. Bonner III, Dropwise condensation life testing of self assembled monolayers, in: Proceedings of the 14th International Heat Transfer Conference, Washington, D.C., 2010, pp. 221-226.

[5] H. Zhao, D. Beysens, From droplet growth to film growth on a heterogeneous surface: Condensation associated with a wettability gradient, Langmuir, 11 (2) (1995) 627-634.

[6] K.K. Varanasi, M. Hsu, N. Bhate, W. Yang, T. Deng, Spatial control in the heterogeneous nucleation of water, Applied Physics Letters, 95 (9) (2009) 094101094101-3.

[7] T.S. Leu, H.W. Lin, T.H. Wu, Enhancement of phase change heat transfer by using surface energy patterning techniques, in: Proceedings of the 1st IEEE International Conference on Nano/Micro Engineered and Molecular Systems, Zhuhai, China, 2006, pp. 994-998.

[8] A. Leipertz, A.P. Fröba, Improvement of condensation heat transfer by surface modification, in: Proceedings of the Seventh ASME, Heat and Mass Transfer Conference, Guwahati, India, 2006, pp. K85-K89.

[9] M. M. Derby, A. Chatterjee, Y. Peles and M. K. Jensen, M.M. Derby, A. Chatterjee, Y. Peles, M.K. Jensen, Flow condensation heat transfer enhancement in a minichannel with hydrophobic and hydrophilic patterns, International Journal of Heat and Mass Transfer, submitted for publication.

[10] A. Chatterjee, Vapor space condensation with surface modifications, $\mathrm{PhD}$ thesis, 
Rensselaer Polytechnic Institute, Troy, NY, 2013.

[11] M.A. Kedzierski, J.L. Worthington III, Design and machining of copper specimens with micro holes for accurate heat transfer measurements, Experimental Heat Transfer, 6 (4) (1993) 329-344.

[12] J.L. McCormick, E. Baer, On the mechanism of heat transfer in dropwise condensation, Journal of Colloid Science, 18 (3) (1963) 208-216.

[13] M.H. Grooten, C.W. Geld, The importance of drainage in dropwise condensation from flowing air-steam mixtures, in: Proceedings of the 14th International Heat Transfer Conference, Washington, D.C., 2010, pp. 51-60.

[14] A. Chatterjee, M.M. Derby, Y. Peles, M.K. Jensen, Vapor Space Condensation with Surface Modifications, International Journal of Heat and Mass Transfer, 66 (2013) 889-897.

[15] J.W. Rose, Dropwise condensation theory and experiments: A review, Journal of Power and Energy, 216 (2) (2002) 115-128.

\section{Table Captions}

Table 1: Feature sizes for island-patterns and the distance between the edges of two adjacent hydrophilic circular islands

Table 2: Highest heat transfer coefficients observed for different surfaces

\section{Figure Captions}

Figure 1: Schematic of the loop

Figure 2: Schematic of the vapor chamber.

Figure 3: Patterns of distinct hydrophobic and hydrophilic regions on the $25.4 \mathrm{~mm}$ condensation surface. (a) island-pattern; (b) tree-pattern 
Figure 4: Variation of heat transfer coefficient with inlet vapor velocity for the patterned surface with hydrophilic island diameter $=0.75 \mathrm{~mm}$

Figure 5: (a) Smaller droplets are seen over the hydrophilic regions with feature size of $0.75 \mathrm{~mm}$ when compared to (b) those over the hydrophilic islands of feature size $1.5 \mathrm{~mm}$

Figure 6: Droplets (a) nucleate, (b) grow, (c) coalesce and (d) depart on the patterned surface with hydrophilic island diameter of (i) $1.50 \mathrm{~mm}$ and (ii) $0.75 \mathrm{~mm}$

Figure 7: The highest heat transfer coefficients observed for the patterned condensation surface with various hydrophilic island diameters

Figure 8: The effect of inlet vapor velocity on the heat transfer coefficient for patterned condensation surface with linear and connected hydrophilic regions resembling a tree on an otherwise hydrophobic region; cooling water temperature of $30^{\circ} \mathrm{C}$

Figure 9: A few droplets are observed at the periphery or edge of the condensation surface, but no steady stream of condensate is observed on the tree-pattern; some of the droplets are also seen on the corner of hydrophilic branches and are sucked in to the hydrophilic region

Figure 10: (a) Local view of the bottom-left quarter of the completely hydrophobic surface with bigger droplets and rivulets (b) Local view of the bottom-left quarter of the patterned surface with hydrophilic island diameter of $0.25 \mathrm{~mm}$ dominated by DWC, smaller droplets and no steady rivulets are observed

Figure 11: Comparison between various modified surfaces for the variation of heat transfer coefficients with inlet vapor velocity

\section{Tables}

Table 1: Feature sizes for island-patterns and the distance between the edges of two adjacent hydrophilic circular islands 


\begin{tabular}{|c|c|}
\hline Feature Size (mm) & Distance between Island Edges (mm) \\
\hline $1.50 \mathrm{~mm}$ & $1.45 \mathrm{~mm}$ \\
\hline $0.75 \mathrm{~mm}$ & $0.66 \mathrm{~mm}$ \\
\hline $0.50 \mathrm{~mm}$ & $0.42 \mathrm{~mm}$ \\
\hline $0.25 \mathrm{~mm}$ & $0.20 \mathrm{~mm}$ \\
\hline
\end{tabular}

Table 2: Highest heat transfer coefficients observed for different surfaces

\begin{tabular}{|l|l|}
\hline \multicolumn{1}{|c|}{ Condensation Surface } & \multicolumn{1}{|c|}{$\begin{array}{c}\text { Highest Observed Heat } \\
\text { Transfer Coefficient }\left(\mathbf{W} / \mathbf{m}^{2} \mathbf{K}\right)\end{array}$} \\
\hline Completely hydrophilic & 12400 \\
\hline Completely hydrophobic & 31500 \\
\hline Hydrophilic pattern diameter $\approx 1.50 \mathrm{~mm}$ & 22100 \\
\hline Hydrophilic pattern diameter $\approx 0.75 \mathrm{~mm}$ & 25900 \\
\hline Hydrophilic pattern diameter $\approx 0.50 \mathrm{~mm}$ & 28800 \\
\hline Hydrophilic pattern diameter $\approx 0.25 \mathrm{~mm}$ & 33900 \\
\hline Tree-pattern & \\
\hline
\end{tabular}

\section{Figures}



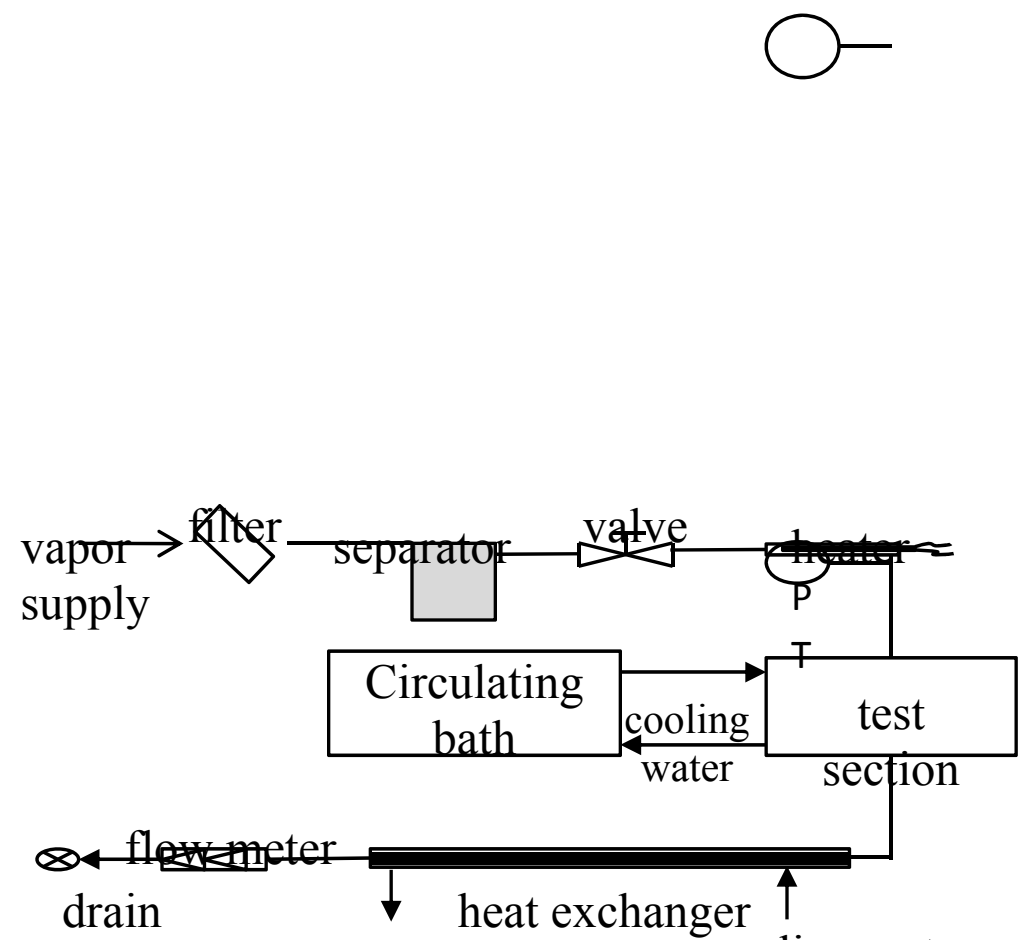

Figure 1: Schematic of the loop cooling water 


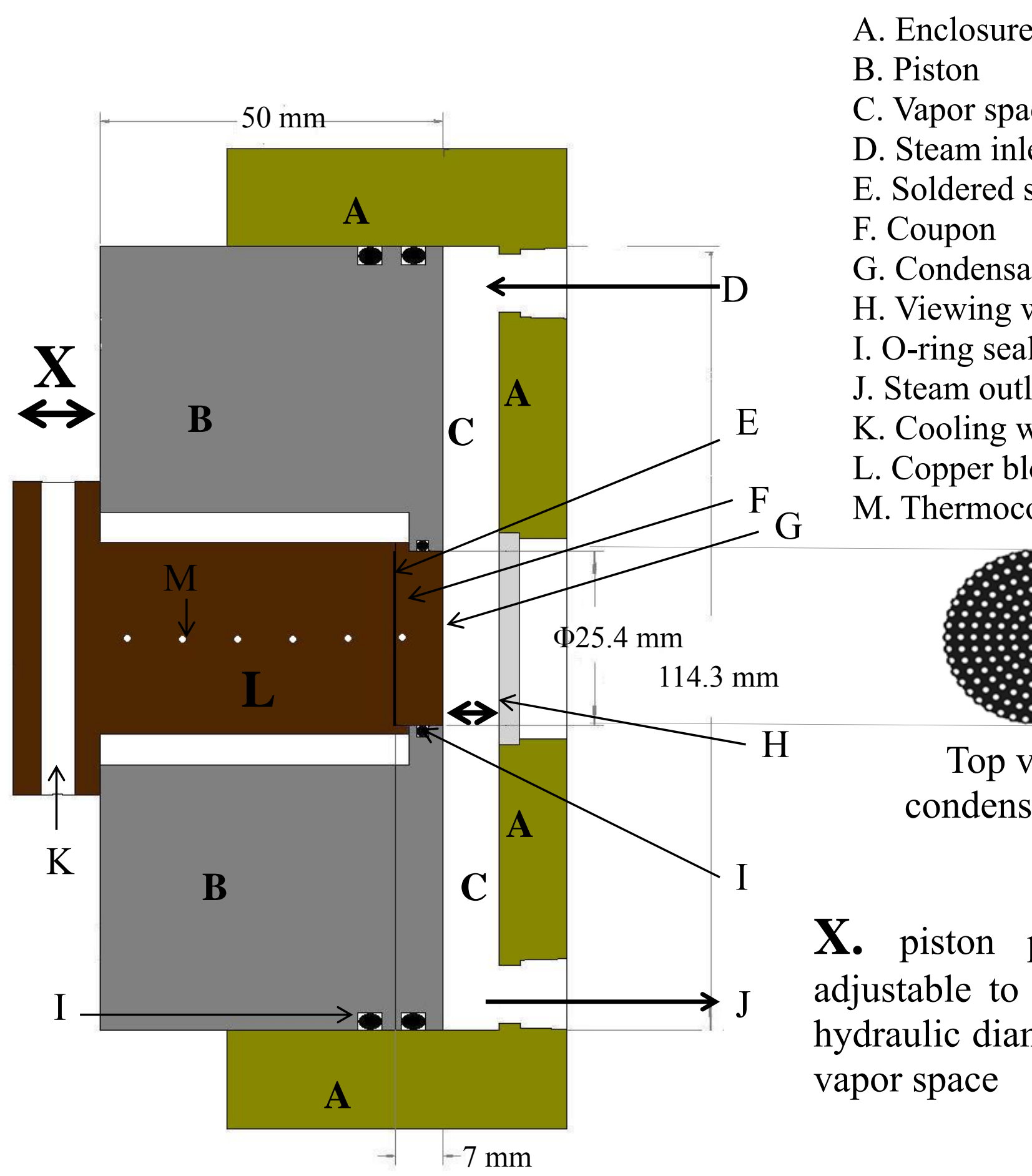

Figure 2: Schematic of the vapor chamber

hydrophobic region

hydrophilic region 


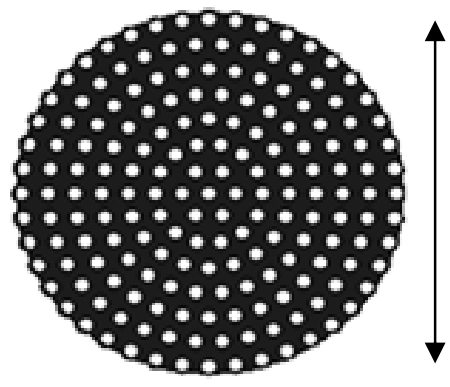

(a)

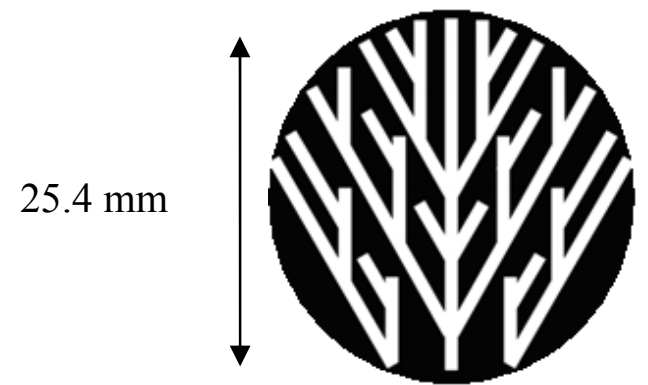

(b)

Figure 3: Patterns of distinct hydrophobic and hydrophilic regions on the $25.4 \mathrm{~mm}$ condensation surface. (a) island-pattern; (b) tree-pattern

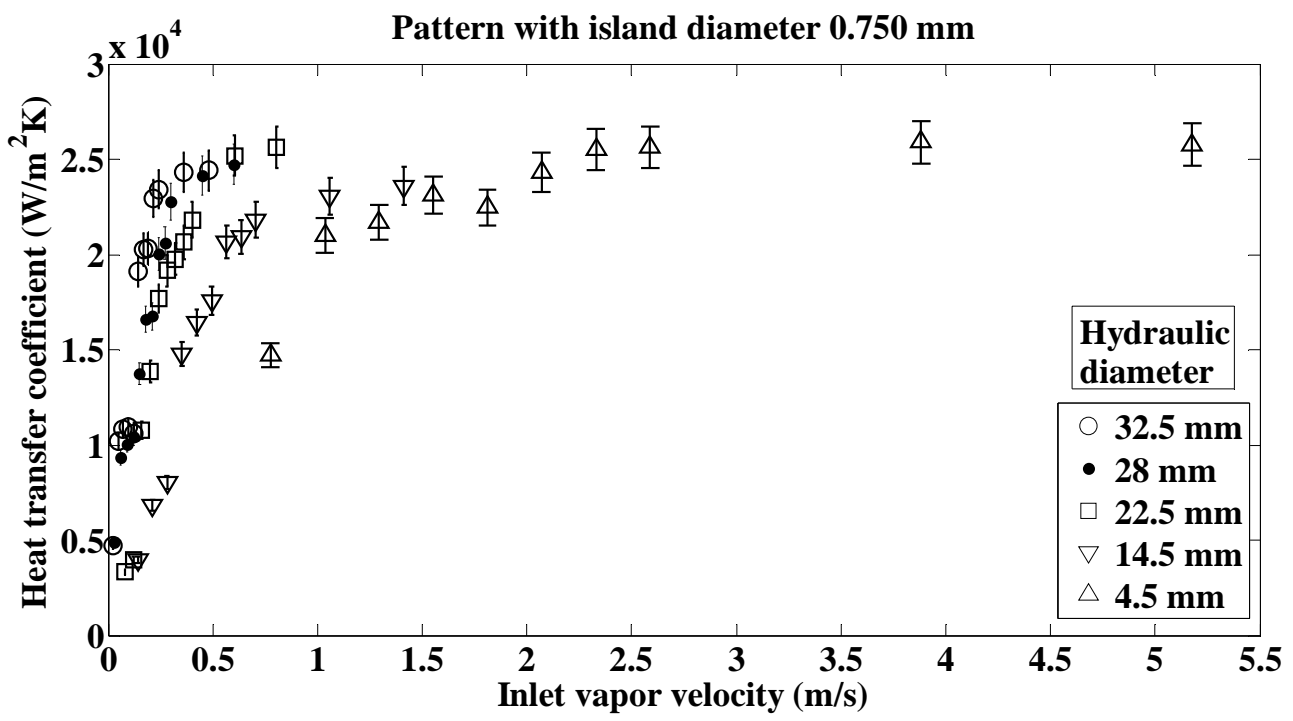

Figure 4: Variation of heat transfer coefficient with inlet vapor velocity for the patterned surface with hydrophilic island diameter $=0.75 \mathrm{~mm}$ 


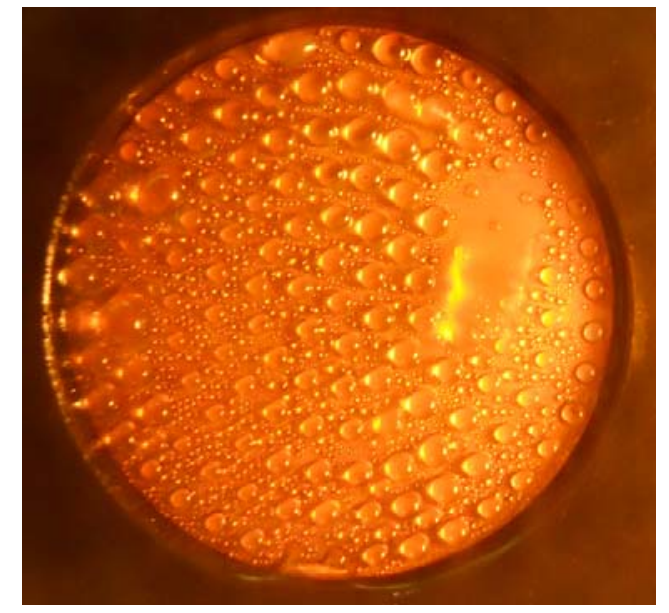

(a)

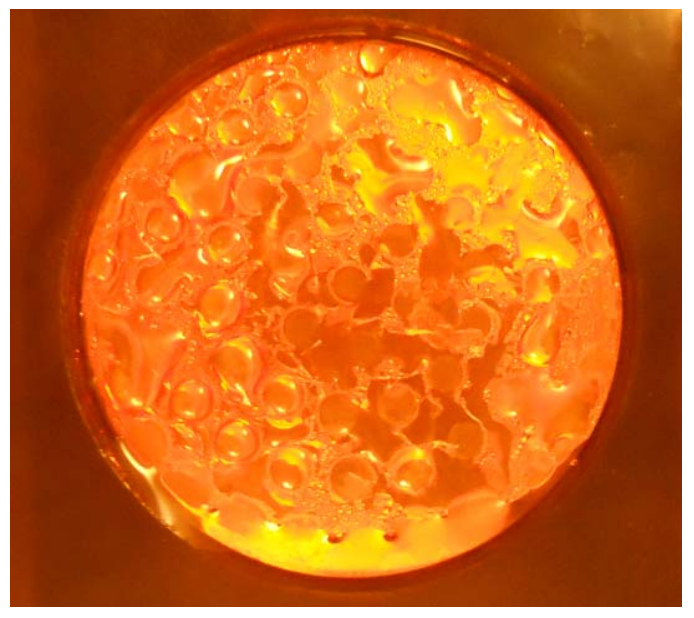

(b)

Figure 5: (a) Smaller droplets are seen over the hydrophilic regions with feature size of

$0.75 \mathrm{~mm}$ when compared to (b) those over the hydrophilic islands of feature size $1.5 \mathrm{~mm}$ 


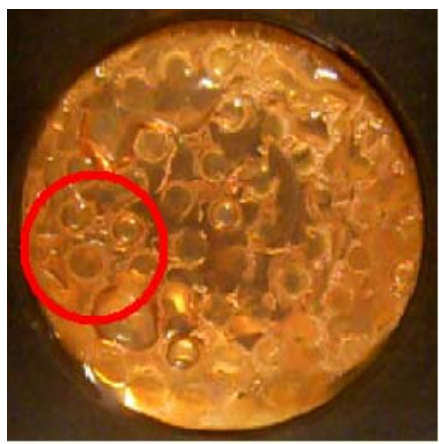

(a) $\mathrm{t}=0 \mathrm{~s}$

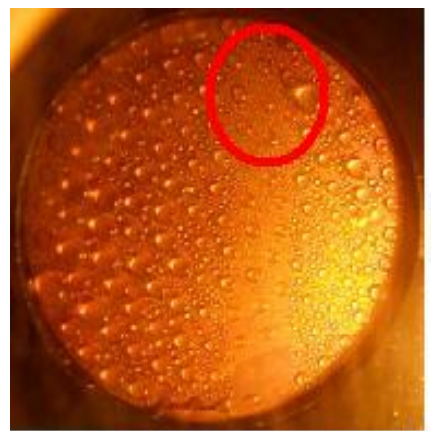

(a) $\mathrm{t}=0 \mathrm{~s}$

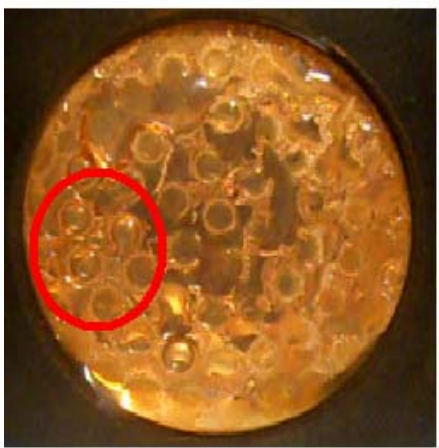

(b) $t=2 s$

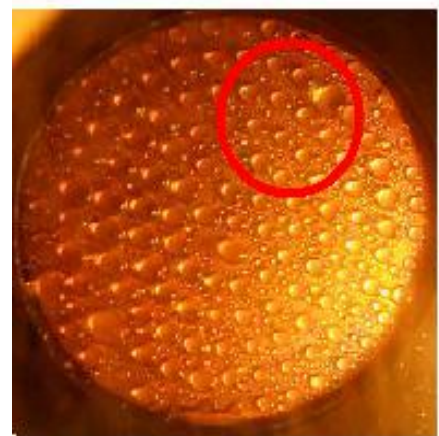

(b) $t=4 \mathrm{~s}$

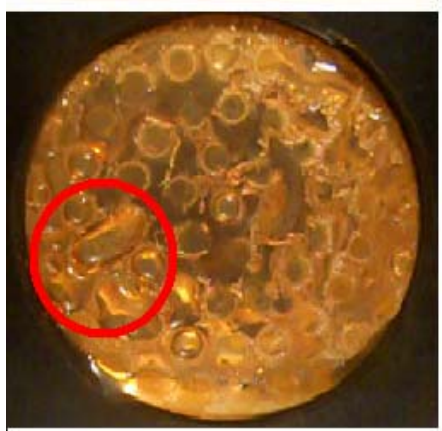

(c) $t=9 s$

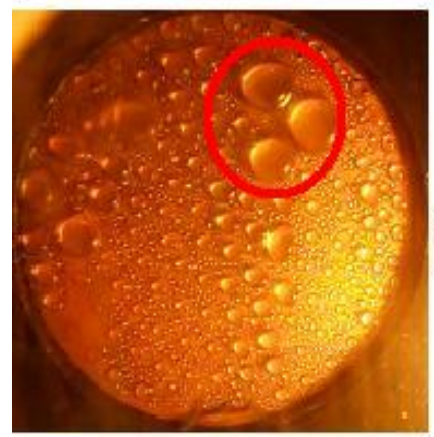

(c) $t=7 s$

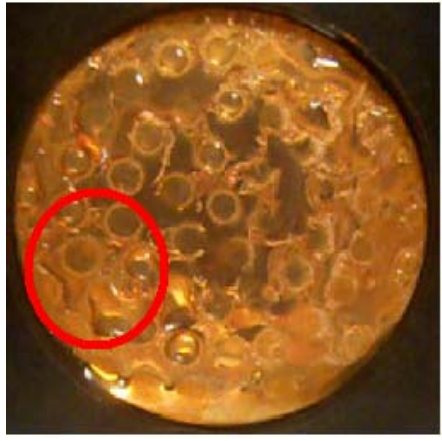

(i) (d) $\mathrm{t}=12 \mathrm{~s}$

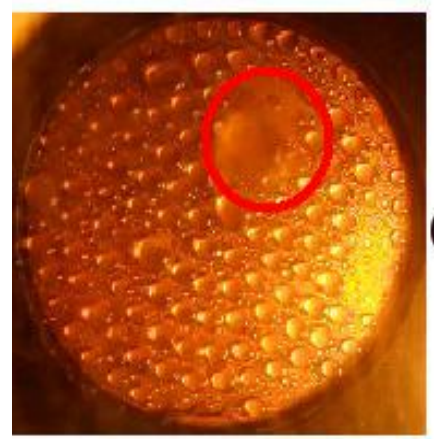

(d) $t=9 s$

Figure 6: Droplets (a) nucleate, (b) grow, (c) coalesce and (d) depart on the patterned surface with hydrophilic island diameter of (i) $1.50 \mathrm{~mm}$ and (ii) $0.75 \mathrm{~mm}$ 


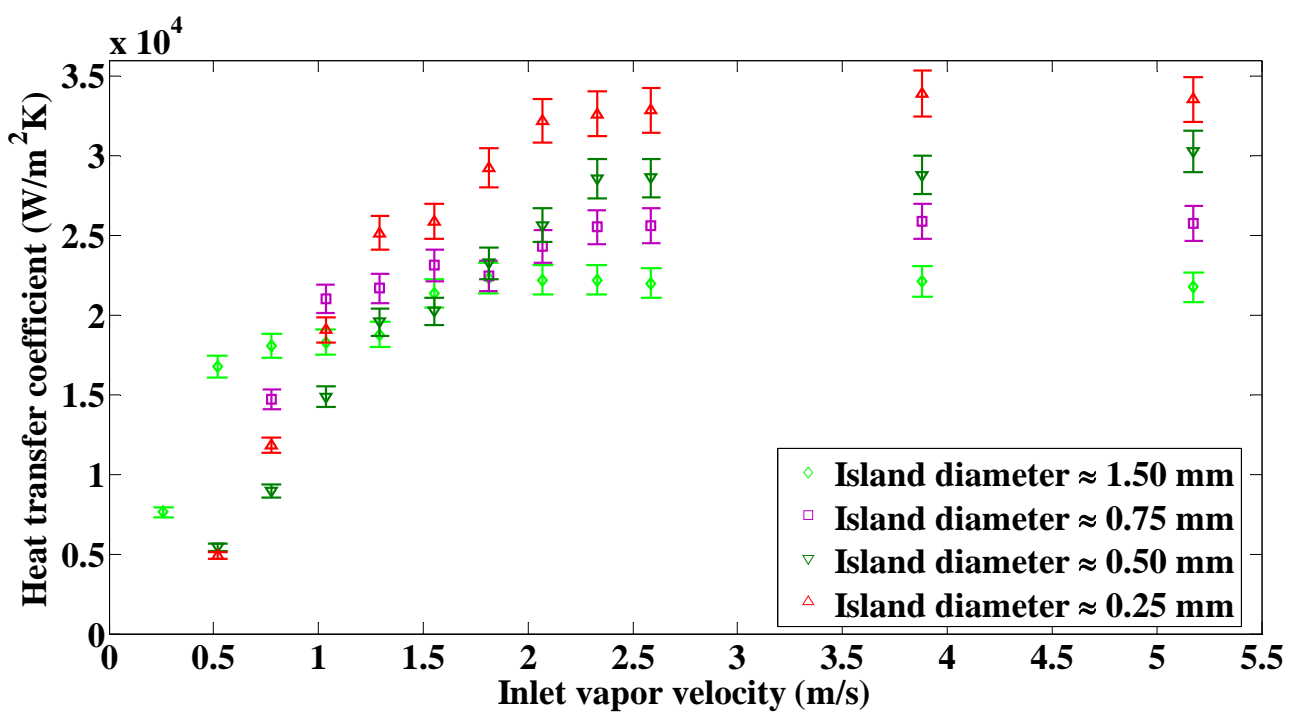

Figure 7: The highest heat transfer coefficients observed for the patterned condensation surface with various hydrophilic island diameters

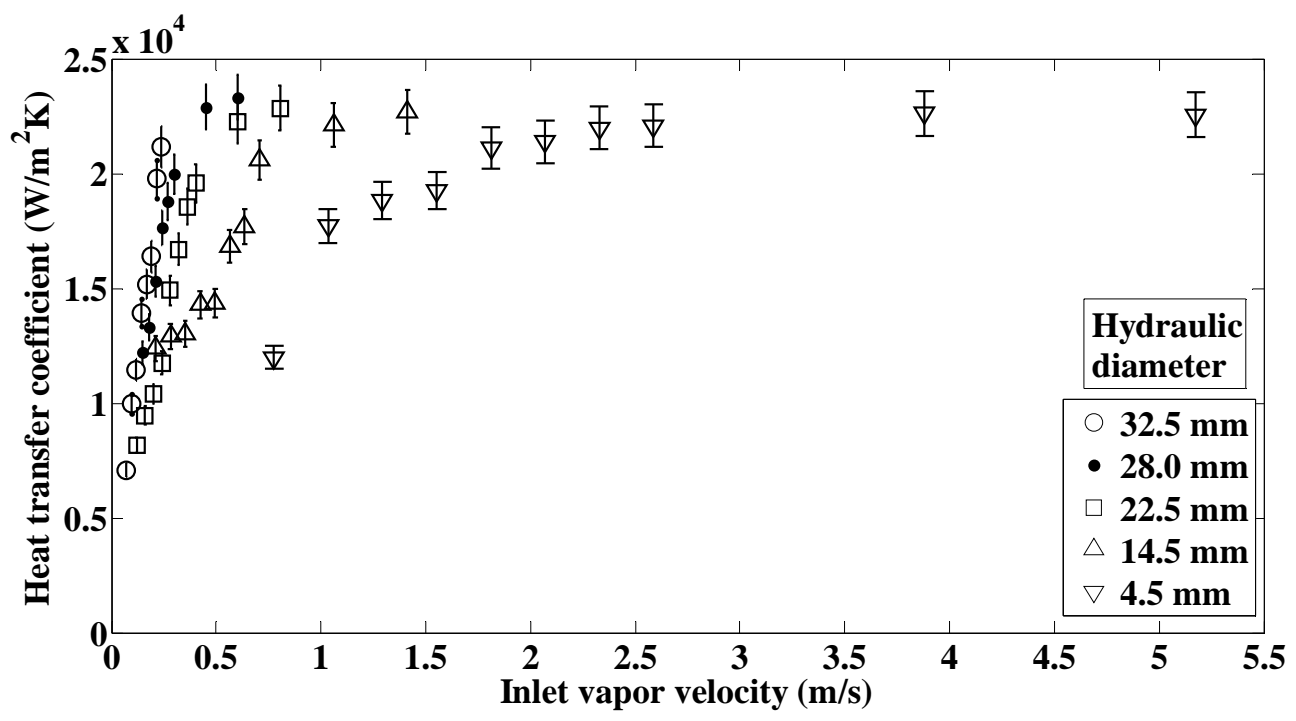


Figure 8: The effect of inlet vapor velocity on the heat transfer coefficient for patterned condensation surface with linear and connected hydrophilic regions resembling a tree on an otherwise hydrophobic region; cooling water temperature of $30^{\circ} \mathrm{C}$

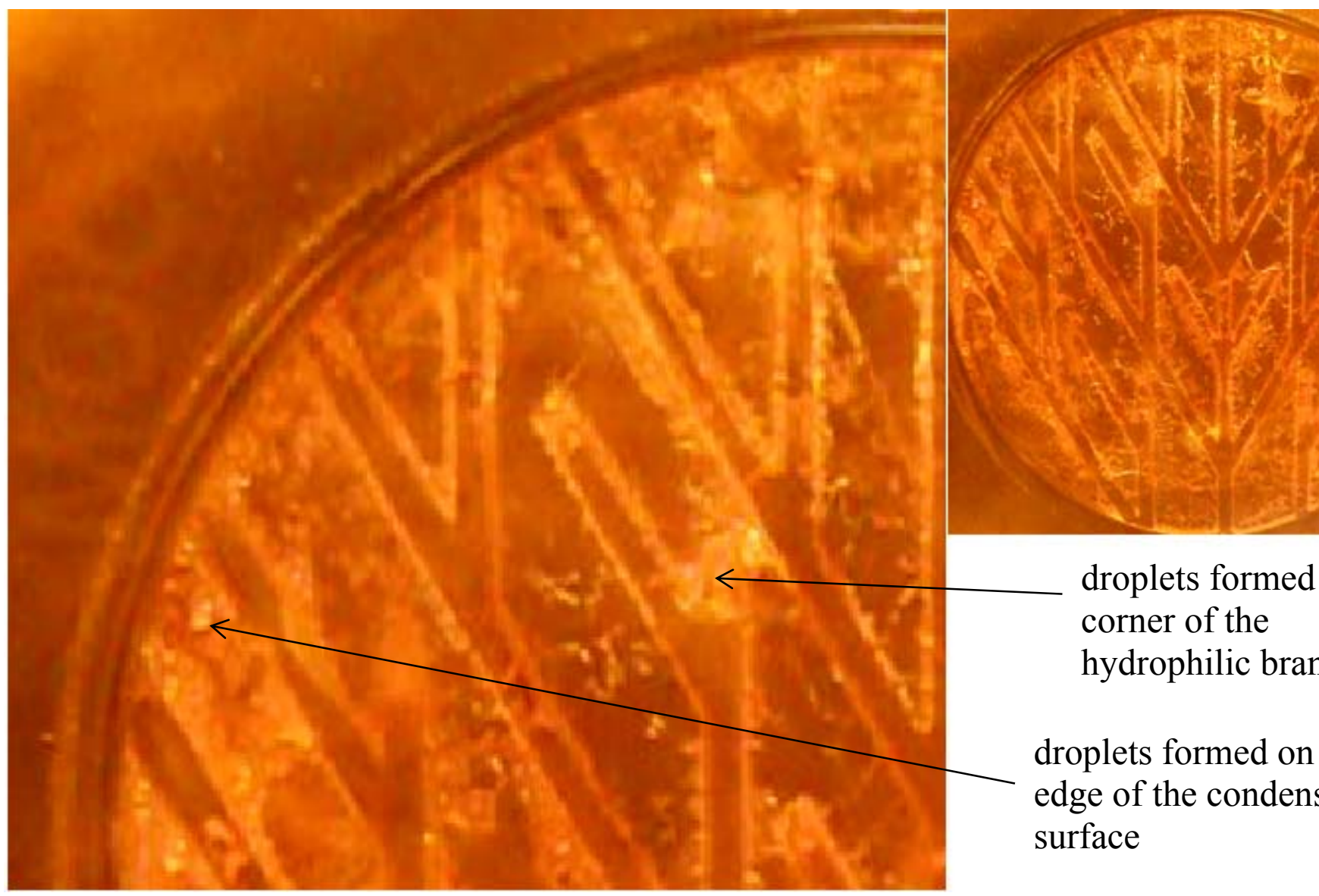

Figure 9: A few droplets are observed at the periphery or edge of the condensation surface, but no steady stream of condensate is observed on the tree-pattern; some of the droplets are also seen on the corner of hydrophilic branches and are sucked in to the hydrophilic region 


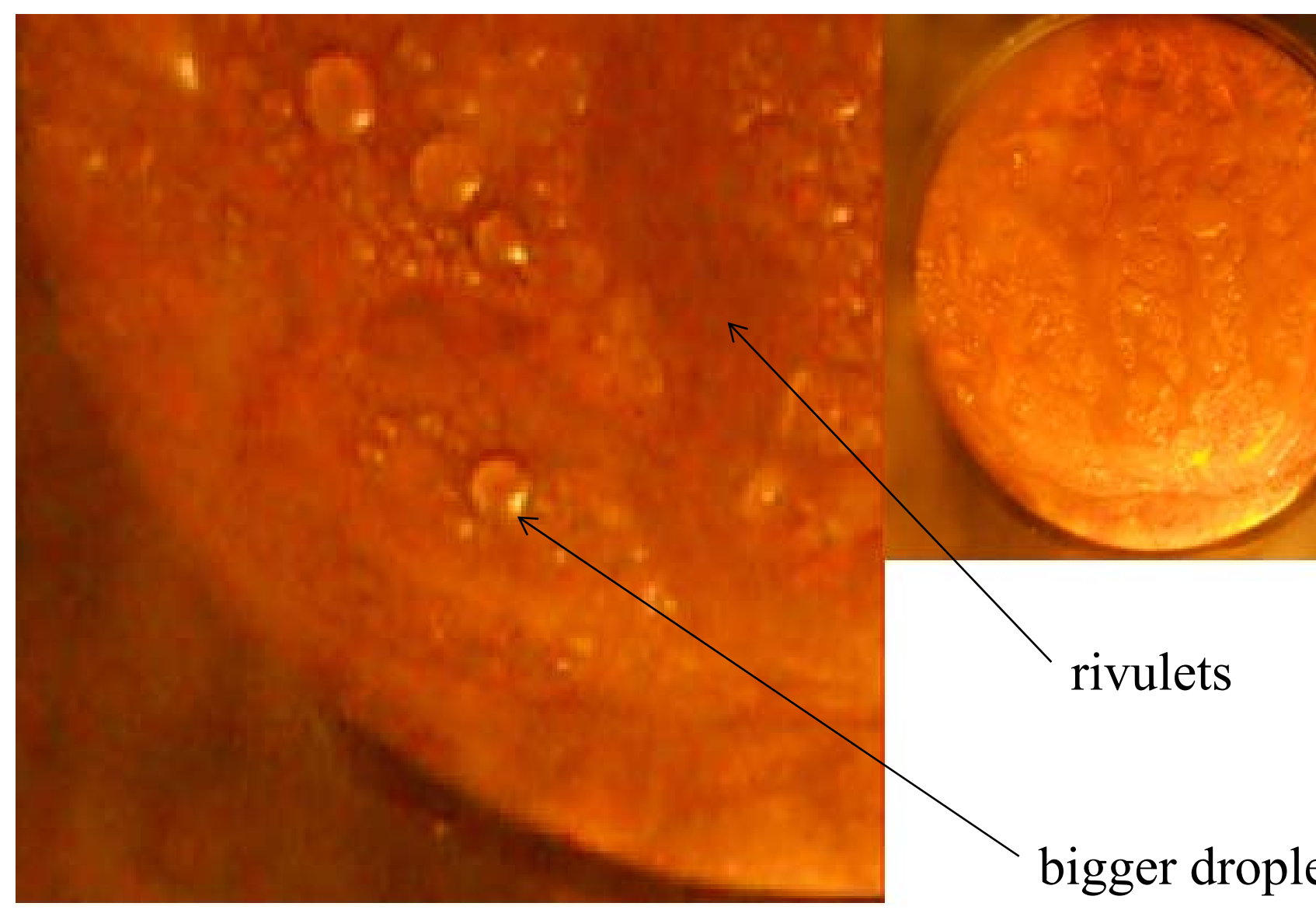

(a) 


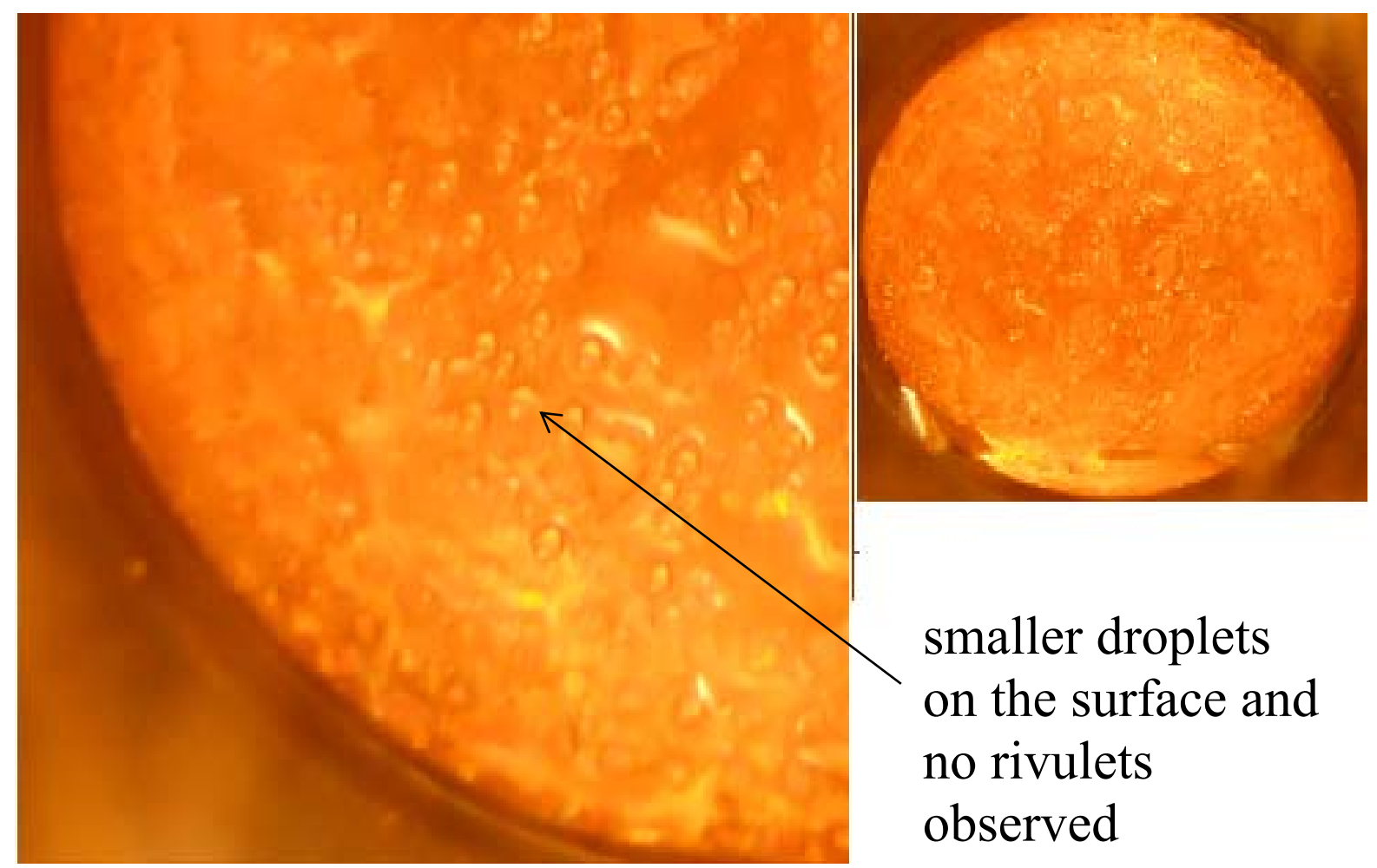

(b)

Figure 10: (a) Local view of the bottom-left quarter of the completely hydrophobic surface with bigger droplets and rivulets (b) Local view of the bottom-left quarter of the patterned surface with hydrophilic island diameter of $0.25 \mathrm{~mm}$ dominated by DWC, smaller droplets and no steady rivulets are observed 
Enhancement of Condensation Heat Transfer with Patterned Surfaces

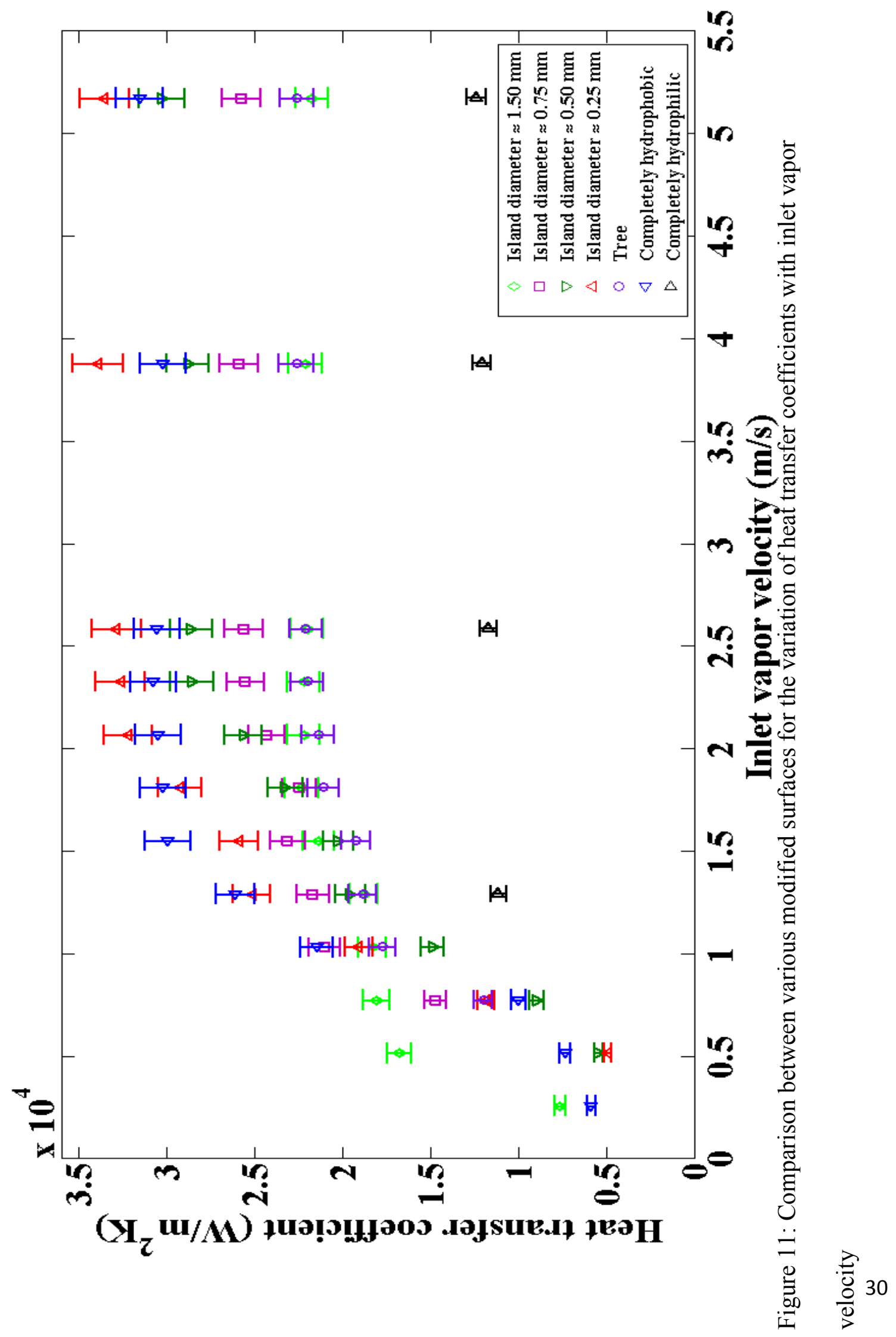

